\title{
A Note on Mitigating the Adverse Scale Effects Associated With Daily Driving Restrictions
}

\author{
Arthur J.Caplan ${ }^{*}$ and Man-Keun Kim
}

December 14, 2017

\begin{abstract}
This paper investigates the role of additional regulation in mitigating the "adverse scale effect" associated with daily driving restrictions, which has become a popular regulatory tool used to control episodic air pollution internationally, especially in developing countries. We find that although an annual vehicle registration tax reduces the incentive to purchase additional vehicles among households whose sole purpose for doing so is to "cheat" the restriction (i.e., the "adverse scale effect"), it does so with an external cost. The cost occurs because households whose purpose for purchasing an additional vehicle is not to cheat the restriction are given the same disincentive with the tax. We show how simple one- and twostage lotteries can be used to not only discriminate between cheater and non-cheater households (in particular, to avoid providing a disincentive to the latter type of household), but also to provide an even stronger disincentive to the former.
\end{abstract}

JEL Classifications: Q52, Q53

Keywords: daily driving restrictions; adverse scale effect; vehicle registration tax; lottery.

\footnotetext{
* Corresponding Author and Professor, Department of Applied Economics, Utah State University, 4835 Old Main Hill, Logan, Utah 84322-4835, arthur.caplan@usu.edu.

${ }^{1}$ Associate Professor, Department of Applied Economics, Utah State University, 4835 Old Main Hill, Logan, Utah 84322-4835, mk.kim@usu.edu.

This research has been funded by Utah Agricultural Experiment Station grant no. UTAO1074.
} 


\section{A Note on Mitigating the Adverse Scale Effects of Daily Driving Restrictions}

\section{Introduction}

Episodic air pollution - in the form of elevated emissions of ozone precursors, carbon

monoxide, or particulate matter from mobile sources - is a recurrent problem in several US cities, as well as in cities throughout the world. ${ }^{2}$ The problem has been pronounced enough over time in the cities of Beijing, China and Mexico City, Mexico to provoke relatively drastic actions on the part of their municipal governments, in particular the imposition of daily driving restrictions aimed at reducing vehicle travel on a year-round basis. Initiated roughly 25 years ago, Mexico City's Hoy no Circula program prohibits households from driving vehicles on assigned days of the week based on the last digit of each vehicle's license plate number (e.g., digits zero and one prohibit driving the vehicle on Mondays, etc.), which translates into a targeted 20 percent removal rate of vehicles on any given week day. Beijing's program, initiated just prior to the Olympic Games in 2008 , has evolved from a roughly 70 percent removal rate to the 20-percent-per-day target of Mexico City's. ${ }^{3}$

Recent empirical studies suggest that neither city's program has met expectations. For example, Eskeland and Feyzioglu (1997) find that gasoline demand in Mexico City had actually increased by seven percent by the time of their study. Davis (2008) also finds an increase in

\footnotetext{
${ }^{2}$ See Cropper et al. (2013), Cummings and Walker (2000), Henry and Gordon (2003), Jiang (2009), Cutter and Neidell (2009), Carnovale and Gibson (2013), Wolff (2014), Gallego et al. (2013a, 2013b), and Osakwe (2010) for examples of previous studies addressing this problem. In a more recent study of driving restrictions implemented in Bogota, Colombia, Zhang et al. (2016) find that restrictions have differential effects on air pollutants because of the pollutants' heterogeneity in the atmospheric chemistry.

${ }^{3}$ Beijing's program is even more restrictive than this. Since 2011 Beijing authorities have rationed license plates themselves, setting a quota on the number of plates issued per year (Guo, 2016; Zhai and Ying, 2016).
} 
gasoline consumption and, along with Gallego et al. (2013a and 2013b), reports an increase in air pollution over the longer term as a result of households having responded by purchasing additional (in most cases older and less-efficient) vehicles in order to "cheat" the daily restrictions. Although the evidence for Beijing is mixed with respect to changes in the concentrations of air pollutants (Wang et al., 2009; Wang et al. 2013; Viard and Fu, 2013; Chen et al., 2013; Cao et al., 2014), the evidence for cheating is consistent with that of Mexico City's (Wang et al., 2013; Chen, 2012; Chen et al., 2013).

This note provides a theoretical underpinning for these empirical results, particularly with respect to the cheating phenomenon described above. Here, we cast this phenomenon in more economic terms by recognizing it as an "adverse scale effect". We derive the necessary condition for an adverse scale effect (ASE) at the household level and leverage this condition to distinguish between ASE and non-ASE households. With respect to regulatory policy aimed at mitigating the ASE, we find that although a vehicle registration tax indeed reduces the incentive to purchase an additional vehicle among households whose sole purpose for doing so is to cheat the restriction (i.e., among ASE households), it does so with an external cost. The cost occurs because households whose purpose for purchasing an additional vehicle is not to cheat the restriction (i.e., non-ASE households) are given the same disincentive as ASE households. We show that one- and two-stage lotteries can be used to not only discriminate between ASE and non-ASE households (in order to avoid providing a disincentive to the latter type of household), but also to provide an even stronger disincentive to the former.

The literature on this particular aspect of the effects associated with a daily driving restriction has thus far been mum. Zhang et al.'s (2016) behavioral model focusses primarily on 
characterizing the extent to which households respond to a restriction by substituting vehicle miles traveled (VMT) across days of the week, rather than on a household's infra-marginal decision of whether to purchase an additional vehicle. They show how the degree to which a household substitutes VMT across restricted and unrestricted days impacts emissions; a relationship we also explore in this paper. Unique to Zhang et al.'s (2016) model is a characterization of the simultaneous effects of inter-day substitution on the emissions of different types of pollutants.

Gallego et al. (2013a) adopt a bundling-model framework to explore the household's inframarginal decision to purchase an additional vehicle or use public transport in response to a driving restriction, which they consider a long-run response. They find that the cost of purchasing and operating an additional vehicle and the extent to which the vehicle will be used during peak and off-peak hours are the key factors in determining the long-run impacts of a driving restriction on emissions. Their empirical analysis of Mexico's Hoy no Circula program suggests that because vehicles represent a "lumpy" or infra-marginal commodity, households who purchased an additional vehicle shortly after implementation of the program were likely close to having made that decision anyway, i.e., they were more likely non-ASE than ASE households, a result that is consistent with our numerical results in Section 4.

The next section lays out a two-day model that characterizes the private benchmark solution as well as the respective substitution effect and ASE that result from a daily driving restriction policy (a more general specification of the model is presented in Appendix A). ${ }^{4}$

\footnotetext{
${ }^{4}$ The household's private decision problem, rather than the social planner's, is the natural benchmark for the ensuing analysis, for reasons stemming from the paper's underlying premise that the daily driving restriction is predetermined, i.e., a fait accompli. We know that as long as the restriction is in place the household's resulting
} 
Section 3 demonstrates how an annual vehicle registration tax levied on an additional vehicle can be used to mitigate the ASE, and further demonstrates the tax's limitation with respect to its associated effect on non-ASE households. In this section we also propose two types of lotteries that overcome this limitation. Section 4 presents a numerical analysis demonstrating the theoretical implications of Sections 2 and 3. Section 5 summarizes and concludes. Appendix B presents the socially efficient solution for the general model presented in Appendix $A$ and demonstrates that a Pigovian tax is both household- and day-specific and can be adjusted for a household's purchase of additional vehicle(s).

\section{A Two-Day Model of Daily Driving Restrictions}

Adopting the example utility specification proposed in Appendix A, we assume the household's welfare function, $u$, is specified as,

$$
u=M+\alpha_{1} \log \left(v_{1}\right)+\frac{\alpha_{2}}{1+v_{1}} \log \left(v_{2}\right)
$$

where $v_{i}$ represents the household's aggregate vehicle miles traveled (VMT) on days $i=1,2, \alpha_{i}$ are corresponding substitution parameters, and $M=\sum_{i=1}^{2} m_{i}$ is aggregate income (numeraire good) defined over the daily income equivalents $m_{i}$. Let the household's daily-equivalent VMT cost functions for days 1 and 2 be written as,

$$
C\left(v_{i}\right)=p v_{i}+0.5 v_{i}^{2}+F_{i}, \quad i=1,2
$$

\footnotetext{
allocation of VMT will generally be socially inefficient as a result of both substitution and scale effects associated with households cheating the restrictions through the purchase of additional vehicles. The pertinent issue that we address here is not the extent to which the household's allocation is socially inefficient, but rather which policies might work best in mitigating the scale effect.
} 
where $p$ represents the household's gas price per VMT and $F_{i}$ represents the daily-equivalent fixed cost of VMT (comprised inter alia of vehicle registration fees and insurance premiums). The household's two-day-equivalent budget constraint is then represented as,

$$
I=M+p\left(v_{1}+v_{2}\right)+0.5\left(v_{1}^{2}+v_{2}^{2}\right)+\left(F_{1}+F_{2}\right) .
$$

Lastly, aggregate emissions for the two days is written as,

$$
E=e\left(v_{1}+v_{2}\right) .
$$

The household's private, two-day decision problem can be written as,

$$
\omega^{P}=\underset{\left\{v_{1}, v_{2}\right\}}{\operatorname{Max}} M+\alpha_{1} \log \left(v_{1}\right)+\frac{\alpha_{2}}{1+v_{1}} \log \left(v_{2}\right) .
$$

Substituting (3) for $M$ in (P1) and differentiating results in optimality conditions,

$$
\begin{aligned}
& \frac{\alpha_{1}}{v_{1}}-\frac{\alpha_{2}}{\left(1+v_{1}\right)^{2}} \log \left(v_{2}\right)-p-v_{1}=0 \\
& \frac{\alpha_{2}}{\left(1+v_{1}\right) v_{2}}-p-v_{2}=0 .
\end{aligned}
$$

For future reference let $v_{i}^{P}, i=1,2$ represent the household's optimal VMT under private decision making for days 1 and 2 .

Assuming its VMT is restricted on day 1 under the daily driving restriction program, i.e., $v_{1} \rightarrow 0$, the household's decision problem is effectively reduced to,

$$
\omega^{R}={ }_{v_{2}}^{\operatorname{Max}} M+\alpha_{2} \log \left(v_{2}\right)
$$

subject to,

$$
I=M+p v_{2}+0.5 v_{2}^{2}+\left(F_{1}+F_{2}\right)
$$

resulting in optimality condition,

$$
\frac{\alpha_{2}}{v_{2}}-p-v_{2}=0
$$

and, solving (6), optimal $v_{2}^{R}$. 
Comparing equations (5b) with (6) we note that $v_{2}^{R}>v_{2}^{P}$ for any $v_{1}^{P}>0$, i.e., the household exhibits a "positive substitution effect" on day 2 as a result of the driving restriction placed on day 1 . To see this, rewrite these two equations as,

$$
\begin{aligned}
& \frac{\alpha_{2}}{v_{2}}=\left(p+v_{2}\right)\left(1+v_{1}\right) \\
& \frac{\alpha_{2}}{v_{2}}=p+v_{2} .
\end{aligned}
$$

Now note that when the $v_{2}^{P}$ solving $\left(5 \mathrm{~b}^{\prime}\right)$ is substituted to $\left(6^{\prime}\right)$ we obtain $\frac{\alpha_{2}}{v_{2}}>p+v_{2}$, which implies that $v_{2}$ must increase to bring equality, in turn implying $v_{2}^{R}>v_{2}^{P}$.

In addition to this substitution effect, a household exhibits an ASE when, as described in Section 1, the household purchases an additional vehicle solely for the purpose of circumventing its daily driving restriction, i.e., solely for the purpose of being able to drive on what would otherwise be a restricted day of the week. In this way, the ASE household "cheats" its restriction once the restriction is imposed. To the contrary, a non-ASE household purchases an additional vehicle for reasons other than to circumvent its daily driving restriction, i.e., not to cheat its restriction once the restriction is imposed. For example, the motive for a non-ASE household's purchase would purely be to provide a newly licensed family member with access to an additional vehicle, irrespective of which days of the week that vehicle is permitted to be driven a priori. ${ }^{5}$

Here, we derive the necessary condition for a household to exhibit an ASE. In doing so, we ultimately define an ASE household's "expected net infra-marginal benefit" associated with the

\footnotetext{
${ }^{5}$ We obviously abstract from reality here, as some households could purchase an additional vehicle to "partially cheat" the restriction, i.e., to sometimes circumvent the restriction and sometimes not. Allowing for partial cheating in the context of this model would unnecessarily complicate the ensuing analysis.
} 
purchase of an additional vehicle, both in the absence and presence of additional regulation. By "additional regulation" we mean regulation in addition to the daily driving restriction that has been imposed a priori, e.g., the levying of a registration tax or lottery jointly with the driving restriction. As we will see, the necessary condition depends upon the ASE household's expected net infra-marginal benefit because from the ASE household's perspective the assumed uncertainty about how and whether the purchase of the additional vehicle will ultimately permit it to circumvent the daily driving restriction is unresolved at time of purchase. Uncertainty in this case pertains not only to the chance that the license plate number assigned by the regulator to the additional vehicle will ultimately preclude the household from driving on what is currently already a restricted day of the week, but also to the way in which the household will incorporate the additional vehicle into its fleet. Maintenance of this type of uncertainty in the 'mind' of the household is central to the ensuing analysis of lotteries as potential mechanisms to mitigate the ASE.

Letting $E\left[\omega^{P}\right]$ represent the expected value of $\omega^{P}$ as defined in problem (P1) inclusive of operating an additional vehicle, the necessary condition for an ASE household to purchase an additional vehicle in the absence of additional regulation (undiscounted for simplicity) is,

$$
\begin{aligned}
& E\left[\omega^{P}\right]-\left(A+F^{A}\right) \geq \omega^{R} \\
& \text { or, } \\
& \Omega=E\left[\omega^{P}\right]-\omega^{R}-\left(A+F^{A}\right) \geq 0 .
\end{aligned}
$$

where (i) $A$ is the (two-day equivalent) one-time purchase price of the additional vehicle, and (ii) $F^{A}$ is the household's (two-day equivalent) total fixed cost associated with the additional vehicle. 
As (7) makes clear, in the absence of additional regulation an ASE household ultimately compares its restricted welfare, $\omega^{R}$, with the expected value of circumventing the driving restriction, $E\left[\omega^{P}\right]$, net of the additional vehicle's purchase price and associated fixed costs. The expectation operator on $\omega^{P}$ reflects the fact that an ASE household ultimately envisions being successful in cheating the driving restriction, albeit with the uncertainty associated with having to incorporate the operation of an additional vehicle into its fleet in the process of effectively re-solving its private decision problem (P1). Equation (7) therefore represents a standard comparison between the household's expected net benefit associated with successfully cheating the driving restriction versus the certain net benefit obtained from choosing to continue abiding by the restriction.

Equation (7') expresses this necessary condition in terms of what we call the ASE household's expected net infra-marginal benefit. It then naturally follows that the ASE household's expected net infra-marginal benefit from purchasing an additional vehicle in the presence of additional regulation can be defined as $\Psi(\Omega)$, where $\Psi: \Omega \rightarrow \mathbb{R}$ represents a household-specific regulatory mapping function. The necessary condition for a household to exhibit an ASE in the absence(presence) of additional regulation is can therefore be expressed most succinctly as $\Omega \geq 0(\Psi(\Omega) \geq 0)$. In other words, if a household exhibits an ASE, whether in the absence or presence of additional regulation, then its expected net infra-marginal benefit from purchasing an additional vehicle must be non-negative. ${ }^{6}$ Further, since additional

\footnotetext{
${ }^{6}$ If the ASE household perceives low-enough transaction costs $\left(T^{c}\right)$, e.g., with respect to the time and effort necessary to calculate its $\Omega$ (or $\Psi(\Omega)$, whichever the case may be) as well as locate and purchase an additional vehicle, then the necessary condition for a household to exhibit an ASE also defines the sufficient condition for the household to exhibit an ASE. In this case it is sufficient for an ASE to exist whenever $\Omega-T^{c} \geq$ $0\left(\right.$ or $\left.\Psi(\Omega)-T^{c} \geq 0\right)$.
} 
regulation in the presence of an existing daily driving restriction is presumed to impose additional cost on a household, $\Omega>\Psi(\Omega)$.

Before turning to the discussion of regulatory schemes that might be used to mitigate a household's ASE, we appeal to the definitions above in order to formally distinguish ASE from non-ASE households. ${ }^{7}$ This distinction draws upon the fact that although a non-ASE household bases its decision of whether to purchase an additional vehicle upon the same cost portion of $\Omega$, i.e., the value of $A+F^{A}$, it does not base its decision on the same benefit portion, i.e., $E\left[\omega^{P}\right]-\omega^{R}$. Rather, the non-ASE household's benefit from the additional vehicle is derived solely from the added benefits the household expects to enjoy from that point forward in a post-restriction state of the world as a result of having incorporated the additional vehicle into its existing vehicle fleet.

Specifically, the non-ASE household's net infra-marginal benefit from purchasing an additional vehicle in the absence of additional regulation is defined as $\widehat{\Omega}=E\left[\omega^{R}\right]-\left(A+F^{A}\right)$, where in this case the expectation operator on $\omega^{R}$ reflects the uncertainty associated with having to incorporate the operation of an additional vehicle into its fleet in the process of effectively re-solving its restricted decision problem (P2). Similar to an ASE household, the nonASE household's net infra-marginal benefit in the presence of additional regulation is then defined as $\Psi(\widehat{\Omega})$. Thus, an ASE household bases its decision on whether to purchase an additional vehicle upon the value of $\Omega$ (absent additional regulation) or $\Psi(\Omega)$ (in the presence

\footnotetext{
${ }^{7}$ It is important to note that because the regulator is precluded from distinguishing ASE from non-ASE households ex ante as well as ex post, the regulator is consequently precluded from designing a socially efficient solution to this problem. Therefore, the best a regulator can do is to reduce the incentive any household may have to impose an ASE on society at large.
} 
of additional regulation), while a non-ASE household bases its decision upon $\widehat{\Omega}$ or $\Psi(\widehat{\Omega})$, respectively.

We now turn to an analysis of the potential impacts of an "additional regulation" as described above, in particular the impacts of a lump-sum registration tax levied by an imperfectly informed regulator, where by "imperfectly informed" we mean that the regulator is uninformed about a given household's $\Omega$ or $\widehat{\Omega}$, whichever the case may be. Thus, the regulator is uninformed about the household's status as a potentially ASE or non-ASE household.

\section{Vehicle Tax and Lotteries}

Levying an annual vehicle registration $\operatorname{tax}, T^{R}$, on an ASE-household's additional vehicle causes its associated regulatory mapping function to be specified as

$$
\Psi(\Omega)=E\left[\omega^{P}\right]-\omega^{R}-\left(A+F^{A}+T^{R}\right) .
$$

Clearly, $\frac{\partial \Psi(\Omega)}{\partial T^{R}}<0$, implying that the registration tax can be used by the regulator to reduce a household's ASE. ${ }^{8}$ However, the regulator is confronted with two problems in using the annual tax, stemming from both its imperfect information about household type and the fact that the regulatory mapping function for a non-ASE household results in $\frac{\partial \Psi(\widehat{\Omega})}{\partial T^{R}}<0$ as well. ${ }^{9}$

\footnotetext{
${ }^{8}$ Fullerton and West (2002) find that a vehicle registration tax based on mileage (i.e., annual odometer readings) can mimic a first-best emissions tax under the assumption of identical or non-identical consumers. Registration taxes based vehicle characteristics are shown to be second-best. Innes (1996) shows that an imperfectly informed regulator's optimal emissions control policy includes joint fuel content standards, fuel taxes, and vehicle registration taxes tied to the mileage the regulator anticipates will be driven per vehicle. In a dynamic context, periodic registration taxes are shown to be efficient. Giblin and McNabola (2009) find that vehicle registration taxes in Ireland based upon expected $\mathrm{CO}_{2}$ emissions could result in a roughly $4 \%$ reduction in the emissions over time. Vehicle registration taxes across Europe vary significantly (Kunert and Kuhfeld, 2007), as they do in the US (NCSL, 2016). Several other studies assess the efficacy of vehicle registration taxes in controlling environmental externalities (Ajanovic et al., 2016; Liu and Cirillo, 2015; Feng et al., 2013; Hennessy and Tol, 2011; Mabit and Fosgerau, 2011; Fullerton and West, 2010; Barter, 2005; West, 2004).

${ }^{9}$ This is because $T^{R}$ also appears negatively in the expression for $\Psi(\widehat{\Omega})$.
} 
First, because it is uninformed about the household's $\Omega$ value, the best the regulator can hope to achieve with an annual registration tax is a reduction in the probability of a given ASE household's potential ASE, rather than its ASE per se. Figure 1 depicts a case where the regulator is cognizant of the probability density function associated with a given ASE household's $\Omega$ (assumed normal for sake of example - initially mean-zero). Here, $T^{R}>0$ induces a leftward shift of the ASE household's density function and a corresponding decrease in the density corresponding to $\Omega \geq 0$ equal to area $\mathrm{A}$.

\section{[INSERT FIGURE 1 HERE]}

Second, as a result of the regulator's concomitant inability to distinguish ASE from non-ASE households, the annual registration tax is, by definition, levied indiscriminately across these two types of households. Therefore, because $\frac{\partial \Psi(\widehat{\Omega})}{\partial T^{R}}<0$, the tax also induces a leftward shift of the non-ASE household's density function, resulting in a decrease in the density corresponding to $\widehat{\Omega} \geq 0$ depicted by area A as well. Recall from Section 1 that reducing a non-ASE household's incentive to purchase an additional vehicle should be neither a regulatory goal nor casualty, as these types of households are not expressly attempting to "cheat" the daily driving restriction.

As we now show, one way to solve this second problem is to implement one of a number of possible simple or compound lotteries in conjunction with the annual registration tax. ${ }^{10}$ Here we describe two such lotteries. By investigating the role that lotteries might play in reducing the inefficiency associated with an annual vehicle registration tax, we admittedly eschew the obvious possibility that the regulatory authority might instead implement a fully-informed,

\footnotetext{
${ }^{10}$ For any lottery to work in mitigating an ASE, households must be required to register their additional vehicles prior to the lottery's implementation.
} 
targeted license-plate numbering scheme, whereby the numbers of each of the household's existing license plates are taken into consideration by the regulator (e.g., through access to a database of existing vehicle registrations) before issuing the license plate to the household for its additional vehicle. In this way, the regulator would be able to eliminate the ASE without relying on chance. While true, we proceed with the presumption that a targeted, commandand-control approach of this type is considered to be draconian, unfair, or politically unacceptable. ${ }^{11}$ Although not market-based regulation per se, the lottery, as conceived in this instance, is instead "chance-based" and "eminently fair", similar to how lotteries are often used to ration charter school admissions and wilderness recreation access in the US (Kerr, 1995). ${ }^{12}$ Imposing a lottery $L$ on an ASE household causes the household's associated regulatory mapping function to be specified as,

$$
\Psi(\Omega)=(\Omega \mid L)
$$

\footnotetext{
${ }^{11}$ This said, used car markets in certain Latin American cities enable households searching for second-hand additional vehicles to effectively circumvent their driving restrictions by selecting the license plate numbers along with the vehicles (Cantillo and Ortuzar, 2014). This is potentially a significant factor in undermining the longer-term effects of daily driving restriction regulations on local air pollution since the import of relatively dirty used vehicles from outside a restricted area is likewise a common occurrence (Gallego et al., 2013a, 2013b). As our paper makes clear, the extent to which vehicle owners are able to choose their plate numbers affects the extent to which an ASE is mitigated. If vehicle owners are permitted to choose their plate numbers deterministically, plate assignment will no longer serve as an effective instrument for mitigating the ASE. Alternatively stated, the uncertainty associated with drivers being precluded from determining their vehicle's plate number (e.g., as a result of a lottery) is crucial for mitigation of the ASE.

${ }^{12}$ The license plate allocation system in Bejing provides another interesting case. Regulators there periodically alter the assignment of daily driving restrictions (eBejing.com, 2015). However, the restrictions are announced to drivers well in advance of taking effect - they occur on a pre-set 13-week rotating basis and are implemented solely for traffic management purposes. The announcements therefore do not incorporate an element of uncertainty and therefore do not mimic a lottery. Granted, if the announcements did occur on a random basis then they would indeed mimic the lotteries that we describe in the paper. But it would seem that the costs associated with this type of surprise (e.g., drivers would be forced to respond with unplanned adjustments to their transportation decisions) outweighs the benefit of mitigating the ASE (presumably drivers would temper their preference for an additional vehicle with the fore-knowledge that assignment of plate numbers will be randomized post-purchase). A negative net benefit is thus the likely reason why periodic alterations to the assignment of digits to days in these cities are not randomized.
} 
where the expression $(\Omega \mid L)$ denotes that the ASE household's expected net infra-marginal benefit from purchasing an additional vehicle is now explicitly conditioned on a specific $L$, which, as we show below, again implies $\Omega>\Psi(\Omega)$.

To the contrary, a non-ASE household's expected net infra-marginal benefit from purchasing an additional vehicle is not conditioned on $L$, reflecting the fact that the motive for a non-ASE household's purchase is not to circumvent the driving restriction and thus its purchase decision is independent of which days of the week that vehicle is permitted to be driven. Therefore, a lottery has no effect on a non-ASE household's expected net infra-marginal benefit, which implies $\Psi(\widehat{\Omega})=\widehat{\Omega}$.

Perhaps the simplest type of lottery to consider is a "single-stage", or simple lottery $L^{S S}$, whereby numbers ending in zero and one restrict the vehicle on Mondays, two and three restrict on Tuesdays, etc. If the regulator were to randomly assign license plate numbers according to this particular method and, for sake of example, the household's VMT is currently restricted solely on Monday of each week, then over the course of a five-day week the household would have an 80 percent chance of receiving a plate number that permits it to drive the additional vehicle on Mondays. In this case, the effect of $\mathrm{L}^{s s}$, which we denote as on the expected infra-marginal benefit of a risk-averse ASE household, is $\Psi(\Omega)=0.8 \Omega<\Omega$. Graphically speaking, the lottery induces a leftward shift of the ASE household's density function (as in Figure 1), but not in a non-ASE household's.

A second type of lottery, henceforth denoted as "two-stage", or compound lottery $\mathrm{L}^{t s}$, is capable of inducing a larger decrease in the ASE household's expected net infra-marginal benefit, while again having no effect on that of a non-ASE household. In the first stage of $\mathrm{L}^{t s}$, 
prior to a household's actual purchase of the additional vehicle, the regulator flips a fair coin. If the coin turns up heads, the household is assured of being issued a license plate for the additional vehicle whose last digit matches that of a current vehicle in its fleet. For example, if a household currently has two vehicles with license plate numbers ending in 1 and 4 (thus restricting one vehicle on Mondays and the other on Wednesdays), the plate number for the additional vehicle is assured of ending in either 1 or 4 as well. Therefore, for households that are unlucky enough to have gotten a heads on the first flip of the coin, the lottery is finished.

Households that were lucky enough to have gotten a tails on the first flip of the coin then move onto the second stage of $\mathrm{L}^{t s}$, where the regulator again flips a fair coin prior to a household's actual purchase of the additional vehicle. If the coin comes up heads, the household is again assured of being issued a license plate for the additional vehicle whose last digit matches that of a current vehicle in its fleet. If the coin comes up tails, the regulator instead randomly selects the household's license plate number, just as was done using lottery $\mathrm{L}^{S S}$. In the end, therefore, the effect of $\mathrm{L}^{t s}$ on the expected net infra-marginal benefit of a riskaverse ASE household is now $\Psi(\Omega)=0.2 \Omega<\Omega$, which represents a far larger leftward shift of the risk-averse ASE household's density function than that depicted in Figure 1 for lottery $L^{s S}{ }^{13}$

Two implications of this two-stage lottery bear mention. First, getting a heads in either the first- or second-stage coin flip requires the regulator to use information about a household's existing vehicle-registration information in what - as mentioned above - may be considered a draconian or politically unpalatable way before issuing the license plate to the household for its

\footnotetext{
${ }^{13}$ Because the coin flips are independent events, the household's 50 percent chance on the first flip, 50 percent chance on the second flip, and then the 80 percent chance in the final round of the lottery translates into a reduction factor on the risk-averse ASE household's expected net infra-marginal benefit of 20 percent.
} 
additional vehicle. Second, the extent to which a given lottery mimics the fully-informed, targeted license-plate numbering scheme depends directly upon the randomizing mechanism used. For example, if the regulator uses a roll of a die rather than a flip of a coin to determine the probability that a given household advances through the second stage of the lottery to the final, random selection of its license plate number, the household's chance of advancing that far in the process could be greatly diminished. In the limit, the regulator can effectively implement a degenerate lottery, where the probability that the ASE household is able to circumvent its driving restriction is zero. But then the question of political acceptability of such a lottery looms large.

\section{Numerical Analysis}

To demonstrate the two-day model's implications described in Sections 2 and 3, we appeal to numerical analysis. ${ }^{14}$ Parameter values for this analysis are listed in Table 1 and solution values for the private and restricted models are presented in Table $2 .^{15}$

\section{[INSERT TABLES 1 AND 2 HERE]}

From Table 2 we first note that, as expected, $v_{2}^{R}>v_{2}^{P}$. Further, $v_{1}^{P}+v_{2}^{P}>v_{1}^{R}+v_{2}^{R} \Rightarrow$ $E^{P}>E^{R}$, which indicates that the driving-restriction regulation is "effective". For simplicity, and without loss of generality, we henceforth assume that $\omega^{P}=E\left[\omega^{P}\right]$ and $\omega^{R}=E\left[\omega^{R}\right]$. Using the corresponding values in Tables 1 and 2, and again assuming a one-year time horizon

\footnotetext{
${ }^{14}$ We use GAMS v. 23.8 .2 x86_64/MS Windows for this numerical exercise.

${ }^{15}$ Recall that parameters $p$ and $F_{i}, i=1,2$ represent per-VMT gas price and daily-equivalent fixed costs; hence their relatively low values for this analysis. Because our numerical example is solely for illustrative purposes we make no claim that the parameter values, and hence solution values, are representative of any particular realworld case. It is interesting to note that for this particular numerical exercise $\alpha_{1} \approx 5.54$ is the threshold below which $v_{1}^{P}=0$, i.e., where the optimal solution is at the corner for day 1 . We have chosen $\alpha_{1}=7$ in our analysis merely for convenience.
} 
for simplicity, the household's $\Omega$-value reduces to $(1.30-\mathrm{A})$, where again $\mathrm{A}$ represents the one-time, two-day equivalent purchase price of the additional vehicle. Hence, the household satisfies the necessary condition for being an ASE household in the absence of additional regulation for any $\mathrm{A} \leq 1.30 .{ }^{16}$ In the presence of additional regulation the threshold value for $\mathrm{A}$ is reduced according to the regulatory mapping function $\Psi: \Omega \rightarrow \mathbb{R}$ described in Section 3 .

For example, suppose the additional regulation is an annual vehicle registration tax set equal to 0.05 . In this case $\Psi(\Omega)$ reduces to $(1.25-\mathrm{A})$ and the necessary condition for being an ASE household in the presence of the tax is now satisfied for any A $\leq 1.25$. Further, $\Omega>\Psi(\Omega)$ also holds for any A. By way of comparison, a similarly risk-averse non-ASE household's $\widehat{\Omega}$ and $\Psi(\widehat{\Omega})$ values equal $(102.83-\mathrm{A})$ and $(102.78-\mathrm{A})$, respectively, and therefore $\widehat{\Omega}>\Psi(\widehat{\Omega})$ as well for any A. Taken together, these results demonstrate one of the key results of Section 3, namely that an annual vehicle registration tax indiscriminately reduces the incentives of both ASE and non-ASE households to purchase an additional vehicle, in particular both $\Omega \geq \Psi(\Omega)$ and $\widehat{\Omega} \geq \Psi(\widehat{\Omega})$ in the presence of the tax. They also lend some support to Gallego et al.'s (2013a) hypothesis mentioned in Section 1 that in response to the implementation of Mexico City's Hoy no Circula program households purchasing additional vehicles were likely close to having made the purchase regardless, i.e., they were behaving more like the non-ASE households described in this paper. This is because the non-ASE's upper-bound purchase price for an additional vehicle (102.83) is so much larger than that for the ASE household (1.30).

\footnotetext{
${ }^{16}$ Recall that our numerical example is purely for illustrative purposes. Thus any solution value, such as the threshold value for $\mathrm{A}$ in this case, should be considered relative in size to the parameter values chosen for the analysis. Also, recall that all variable values are in daily or two-day equivalents, whichever the case may be.
} 
The main result of Section 3 is also now evident. If the household is an ASE household, then because lottery $L$ reduces the household's $\Omega$-value we again obtain $\Omega>\Psi(\Omega)$. For example, suppose lottery $\mathrm{L}^{S S}$ is adopted by the regulatory authority. Then $\Omega=(1.30-\mathrm{A})>\Psi(\Omega)=$ $0.8(1.30-\mathrm{A})$. For lottery $\mathrm{L}^{t s}, \Omega=(1.30-\mathrm{A})>\Psi(\Omega)=0.2(1.30-\mathrm{A})$. Interestingly, the lotteries do not reduce the threshold level of A, as occurs with a registration tax. As described in Section 3, because the non-ASE household's expected net infra-marginal benefit is independent of the lottery, $\Psi(\widehat{\Omega})=\widehat{\Omega}=(102.83-\mathrm{A})$.

\section{Summary and Conclusions}

Daily driving restrictions, although currently a popular form of regulation used to control episodic air pollution problems worldwide, is nevertheless a second-best approach. Both the substitution and adverse scale effects derived and characterized in this paper attest to the inherent shortcomings of this type of restriction. In addition to characterizing these effects, this paper investigates the efficacy of two forms of regulation - vehicle registration taxes and lotteries - in mitigating the scale effect when the regulator has incomplete information about the households it is tasked to regulate.

We find that although a vehicle registration tax indeed reduces the incentive to purchase additional vehicles among households whose sole purpose for doing so is to "cheat" the restriction (which we have named an "adverse scale effect"), it does so with an external cost. The cost occurs because households whose purpose for purchasing an additional vehicle is not to cheat the restriction are given the same disincentive with a registration tax. We show that one- and two-stage lotteries can be used to not only discriminate between cheater and noncheater households, but also to provide an even stronger disincentive to the former. 
Clearly, no single policy (barring a household- and day-specific Pigovian tax as derived in Appendix B) is capable of controlling episodic pollution at its socially efficient level. Thus, in a practical sense, multiple policy instruments are likely necessary - perhaps quantity regulations such as daily driving restrictions - accompanied by vehicle registration taxes and lotteries to mitigate accompanying scale effects - and VMT or perhaps gas taxes to control for substitution effects. At the very least, it helps to know the limitations associated with a given instrument. This paper has brought to light the fundamental limitations of a registration tax in the presence of daily driving restrictions, and demonstrated how a policy as simple as flipping a coin can be used to address them. 


\section{Appendix A - Generalized Model of the Adverse Scale Effect}

Following Zhang et al. (2016), let $v_{i j}$ represent household $i$ 's total vehicle miles traveled (VMT)

- based on the use of all vehicles in its fleet - on weekday $j=1, \ldots, 5$, of week $w=1, \ldots, 52$, in year $y$ (subscripts $w$ and $y$ will henceforth distinguish variables solely when necessary), and vector $v_{i}=\left(v_{i 1}, \ldots, v_{i 5}\right)$ represent $i^{\prime}$ s associated schedule of daily VMT per week. ${ }^{17}$ Assuming quasilinear preferences, $i$ 's weekly utility function is expressed as $U_{i}=\sum_{j} m_{i j}+B_{i}\left(v_{i}\right), i=$ $1, \ldots, N$, where $m_{i j}$ and $B_{i}\left(v_{i}\right)$ are $i$ 's daily-equivalent numeraire and weekly benefit from VMT, respectively. We adopt standard curvature conditions for $B_{i}\left(v_{i}\right): \frac{\partial B_{i}\left(v_{i}\right)}{\partial v_{i j}}>0, \frac{\partial^{2} B_{i}\left(v_{i}\right)}{\partial v_{i j}{ }^{2}} \leq 0$, as well as $\frac{\partial^{2} B_{i}\left(v_{i}\right)}{\partial v_{i j} \partial v_{i k}} \leq 0, j, k=1, \ldots, 5$ and $j \neq k$ and $\frac{\partial^{2} B_{i}\left(v_{i}\right)}{\partial v_{i j} \partial v_{i k}}<0$ for at least one $j \neq k$. For example, $B_{i}\left(v_{i}\right)=\sum_{j}\left(\frac{\alpha_{i j}}{1+v_{i, j-1}}\right) \log v_{i j}, \alpha_{i j}>0 \forall i, j$, and $v_{i, j-1}=0$ for $j=1$ would be one possible specification for $B_{i}\left(v_{i}\right)$, in which case the marginal utility of VMT on any given day is weighted according to the VMT of the preceding day, with the weight assigned to VMT on day 1 (e.g., Monday) normalized to $\alpha_{i j}$.

The two latter conditions on function $B_{i}\left(v_{i}\right)$ imply that when $i$ 's VMT is reduced on a given day $k$ due to an existing driving restriction on that day, the marginal value associated with its VMT on any other given (unrestricted) day $j$ of the week does not decrease, and it increases for at least one day $j$, all else equal. This condition reflects the fact that household $i$ may potentially adjust its VMT on any given day $j$ in order to accomplish what the reduced amount

\footnotetext{
${ }^{17}$ In concert with the actual programs mentioned in Section 1, we assume that driving restrictions are imposed solely during the weekdays, i.e., Monday through Friday.
} 
of VMT on day $k$ would have otherwise accomplished that day, thus making VMT on day $j$ more valuable. $^{18}$

Let $C_{i}\left(v_{i j}\right)=c_{i}^{g} v_{i j}+c_{i}\left(v_{i j}\right)+F_{i j}$ represent $i$ 's daily-equivalent VMT cost function, where (1) $c_{i}^{g}$ indicates $i^{\prime}$ s gas price per VMT (determined jointly by the prevailing price per gallon and some measure of the overall fuel efficiency of $i$ 's vehicle fleet), (2) $F_{i j}=F_{i k} \forall j, k=1, \ldots, 5$ represents $i^{\prime}$ s daily-equivalent fixed cost of VMT, and (3) $c_{i}\left(v_{i j}\right)$ is $i^{\prime}$ s daily-equivalent variable cost of VMT (including depreciation, maintenance expenses, etc., associated with the household's vehicle fleet), with standard curvature conditions $\frac{\partial c_{i}\left(v_{i j}\right)}{\partial v_{i j}}>0$ and $\frac{\partial^{2} c_{i}\left(v_{i j}\right)}{\partial v_{i j}{ }^{2}} \geq 0$. Household $i$ 's weekly budget constraint can therefore be written as $I_{i}=\sum_{j}\left(m_{i j}+C_{i}\left(v_{i j}\right)\right)$, where $I_{i}$ represents $i$ 's constant weekly-equivalent income level. Lastly, let $E_{j}=\sum_{i} e_{i} v_{i j}$ be the community's aggregate daily emissions, where $e_{i}$ represents $i$ 's per-mile emissions factor, which is based upon the type of vehicles in $i^{\prime}$ s fleet and $i^{\prime}$ s driving habits. ${ }^{19}$

Household $i$ 's private, daily decision problem for any given week can therefore be expressed as,

\footnotetext{
${ }^{18}$ The household may choose to respond to a restriction on any given day of any given week in a myriad of ways. For instance, the household may choose to increase its VMT on a set of unrestricted days rather than just one day, or perhaps decrease its VMT on one unrestricted day (e.g., by taking public transport or eliminating a trip altogether) and compensate by increasing its VMT even more on another day. Our cross-partial condition on $B_{i}\left(v_{i}\right)$, i.e., $\frac{\partial^{2} B_{i}\left(v_{i}\right)}{\partial v_{i j} \partial v_{i k}} \leq 0$, does not preclude any such set of responses. It merely states that the marginal value of VMT on any given unrestricted day has increased in response to the imposition of a restriction on any other given day. What ultimately determines a household's optimal response on a weekly basis are therefore the relative values associated with changes in daily VMT. Indeed, it may be optimal for a household to decrease its VMT on an unrestricted day even though the marginal value of VMT on that particular day has increased. This is because the increases in the marginal values of VMT on all of the other unrestricted days exceed that day's by large enough margins.

${ }^{19}$ In a more disaggregated framework, $e_{i}$ might be a weighted average across the types of vehicles in household $i^{\prime}$ s fleet as well as its driving habits for each vehicle.
} 


$$
\omega_{i}^{P}={ }_{v_{i j}}^{\operatorname{Max}} B_{i}\left(v_{i}\right)-\sum_{j}\left(c_{i}^{g} v_{i j}+c_{i}\left(v_{i j}\right)+F_{i j}\right)
$$

resulting in first-order conditions, ${ }^{20}$

$$
\frac{\partial B_{i}\left(v_{i}\right)}{\partial v_{i j}}-c_{i}^{g}-\frac{\partial c_{i}\left(v_{i j}\right)}{\partial v_{i j}} \leq 0 \quad v_{i j}\left[\frac{\partial B_{i}\left(v_{i}\right)}{\partial v_{i j}}-c_{i}^{g}-\frac{\partial c_{i}\left(v_{i j}\right)}{\partial v_{i j}}\right]=0, \quad \forall i, j
$$

which indicates that in its benchmark private decision problem, household $i$ will optimally choose its $v_{i j}$ up to the point where the daily private marginal benefit of VMT is no greater than its corresponding marginal cost for any given week. Henceforth, let $v_{i j}^{P}$ and $E_{j}^{P}$ represent the optimal private levels of daily household VMT and aggregate emissions, respectively, $i=$ $1, \ldots, N, j=1, \ldots, 5$.

Assume household $i$ is assigned certain day(s) of the week, $j \in j^{*}$, where its VMT is restricted. Without loss of generality, we assume this restriction is set such that $v_{i j^{*}}=0, j \in j^{*}$, i.e., the household is precluded from driving on days $j \in j^{*}$. Given this restriction, household $i^{\prime}$ s daily decision problem on unrestricted days of the week for any given week may be expressed as,

$$
\omega_{i}^{R}={ }_{v_{i j}}^{\operatorname{Max}} B_{i}\left(\tilde{v}_{i}\right)-\sum_{j}\left(c_{i}^{g} v_{i j}+c_{i}\left(v_{i j}\right)+F_{i j}\right), j \notin j^{*}
$$

where, for example, $\tilde{v}_{i}=\left(v_{i 1}, v_{i 2}, 0, v_{i 4}, 0\right)$ indicates that VMT restrictions are in effect on days 3 and 5 of each week for household $i$. The resulting first-order conditions for unrestricted days are written as,

$$
\frac{\partial B_{i}\left(\tilde{v}_{i}\right)}{\partial v_{i j}}-c_{i}^{g}-\frac{\partial c_{i}\left(v_{i j}\right)}{\partial v_{i j}}=0, j \notin j^{*}
$$

\footnotetext{
${ }^{20}$ We note that the curvature conditions on $B_{i}\left(v_{i}\right)$ and $c_{i}\left(v_{i j}\right)$ ensure that sufficient second-order conditions for this and all ensuing decision problems are satisfied.
} 
Henceforth, let $v_{i j}^{R}$ and $E_{j}^{R}$ represent the optimal levels of daily household VMT and aggregate emissions, respectively, for the daily driving restriction problem. It is now readily apparent that in the presence of a daily driving restriction, household $i$ exhibits a positive "substitution effect" on at least one of the unrestricted driving days, whereby $v_{i j}^{R}>v_{i j}^{P}$ for that day (or days) $j \notin$ $j^{* 21}$

In other words, the daily driving restriction induces a positive substitution effect on at least one unrestricted day of the week, whereby household $i$ compensates for not being permitted to drive on day(s) $j \in j^{*}$ by driving no less on that unrestricted day $j \notin j^{*}$ (relative to what it otherwise would have driven on that day in the absence of the daily driving restriction). If the driving restriction program is ultimately effective for household $i$ - in the sense that it results in $\sum_{j} v_{i j}^{R}<\sum_{j} v_{i j}^{P}$, which states that household $i$ drives less on a weekly basis in the presence of a daily driving restriction - then the substitution effect alone is not strong enough to completely offset the restriction's effect on household $i^{\prime}$ s total weekly VMT. This would imply $\sum_{i} \sum_{j} v_{i j}^{R}<$ $\sum_{i} \sum_{j} v_{i j}^{P}$ on a community-wide basis if the driving restriction program is effective enough for a requisite number of households, in which case $E_{j}^{R} \gtreqless E_{j}^{P}$ for any $j=1, \ldots, 5$ (because on any given day $v_{i j}^{R}=0$ for some subgroup of households). However, $E_{w}^{R}=\sum_{j} E_{j}^{R}<E_{w}^{P}=\sum_{j} E_{j}^{P}$ as a direct result of $\sum_{i} \sum_{j \notin j^{*}} v_{i j}^{R}<\sum_{i} \sum_{j} v_{i j}^{P}$.

${ }^{21}$ For those readers requiring a formal proof of this result, first apply curvature condition $\frac{\partial^{2} B_{i}(\cdot)}{\partial v_{i j} \partial v_{i k}} \leq 0 \forall j \notin j{ }^{*}, k \in$ $j^{*}$ and note that $\frac{\partial B_{i}\left(\tilde{v}_{i}\right)}{\partial v_{i j}} \geq \frac{\partial B_{i}\left(v_{i}\right)}{\partial v_{i j}}$ for each day $j \notin j^{*}$, i.e., in the presence of a daily driving restriction, the marginal value of VMT on any given non-restricted day does not fall relative to its marginal value in the absence of a daily driving restriction. Next, apply curvature conditions $\frac{\partial^{2} B_{i}(\cdot)}{\partial v_{i j}{ }^{2}} \leq 0$ and $\frac{\partial^{2} c_{i}\left(v_{i j}\right)}{\partial v_{i j}{ }^{2}} \geq 0$, as well as the condition $\frac{\partial^{2} B_{i}\left(v_{i}\right)}{\partial v_{i j} \partial v_{i k}}<$ 0 for at least one $j \neq k$., Equations (A1) and (A2) then imply $v_{i j}^{R}>v_{i j}^{P}$ for that day (or days) $j \notin j^{*}$. 
Similar to the two-day version of the model in the text, letting $E\left[\omega_{i j w}^{P}\right]$ represent the expected value of (the daily-per-week version of) $\omega_{i}^{P}$ as defined above inclusive of operating an additional vehicle, an ASE household $i$ 's expected net infra-marginal benefit from purchasing the additional vehicle in the absence of additional regulation is $\Omega_{i}=$ $\sum_{y=1}^{Y} \beta_{i}^{y-1}\left(\sum_{w} \sum_{j}\left(E\left[\omega_{i j w}^{P}\right]-\omega_{i j w}^{R}\right)\right)-\left(A_{i}+\sum_{y=1}^{Y} \beta_{i}^{y-1}\left(\sum_{w} \sum_{j} F_{i j w}^{A}\right)\right)$, where (1) $\beta_{i}=\frac{1}{1+r}$ represents the household's discount factor based on discount rate $r$ for finite number of years $y=1, \ldots, Y,(2) \omega_{i j w}^{R}$ is the household's optimal net benefit obtained from its restricted decision problem prior to imposition of the driving restriction (derived in Section 3), (3) $A_{i}$ is the one-time purchase price of the additional vehicle, and (4) $F_{i j w}^{A}$ is the household's dailyequivalent fixed cost associated with the additional vehicle.

An ASE household $i$ 's expected net infra-marginal benefit from purchasing an additional vehicle in the presence of additional regulation is then defined as $\Psi_{i}\left(\Omega_{i}\right)$, where $\Psi_{i}: \Omega_{i} \rightarrow \mathbb{R}$ represents a household-specific regulatory mapping function. The necessary condition for a household to exhibit an ASE in the absence(presence) of additional regulation is $\Omega_{i} \geq$ $0\left(\Psi_{i}\left(\Omega_{i}\right) \geq 0\right)$. Further, since additional regulation in the presence of an existing daily driving restriction is presumed to impose additional cost on a household, $\Omega_{i}>\Psi_{i}\left(\Omega_{i}\right), i=1, \ldots, N$. Similar to the discussion in Section 2, a non-ASE household $i$ 's net infra-marginal benefit from purchasing an additional vehicle in the absence of additional regulation is $\widehat{\Omega}_{i}=$ $\sum_{y=1}^{Y} \beta_{i}^{y-1}\left(\sum_{w} \sum_{j} E\left[\omega_{i j w}^{R}\right]\right)-\left(A_{i}+\sum_{y=1}^{Y} \beta_{i}^{y-1}\left(\sum_{w} \sum_{j} F_{i j w}^{A}\right)\right)$. Further, the non-ASE household's net infra-marginal benefit from purchasing an additional vehicle in the presence of additional regulation is defined as $\Psi_{i}\left(\widehat{\Omega}_{i}\right)$. 
Levying an annual vehicle registration tax, $T_{y}^{R}$, on ASE household $i$ causes its associated regulatory mapping function to be specified as $\Psi_{i}\left(\Omega_{i}\right)=\sum_{y=1}^{Y} \beta_{i}^{y-1}\left(\sum_{w} \sum_{j}\left(E\left[\omega_{i j w}^{P}\right]-\right.\right.$ $\left.\left.\omega_{i j w}^{R}\right)\right)-\left(\mathrm{A}_{\mathrm{i}}+\sum_{\mathrm{y}=1}^{\mathrm{Y}} \beta_{\mathrm{i}}^{\mathrm{y}-1}\left(\sum_{\mathrm{w}} \sum_{\mathrm{j}} \mathrm{F}_{\mathrm{ijw}}^{\mathrm{A}}+\mathrm{T}_{\mathrm{y}}^{\mathrm{R}}\right)\right)$ and the results discussed in Section 3 hold in this more general specification as well. Similarly for the single- and two-stage lotteries described in Section 3.

\section{Appendix B - The Social Efficiency Benchmark}

Recalling that $E_{j}=\sum_{i} e_{i} v_{i j}$ represents the community's aggregate daily emissions, where $e_{i}$ represents $i$ 's per-mile emissions factor, let the community's total daily damage function associated with $E_{j}$ be represented by $D_{j}=D\left(E_{j}\right)$, with $\frac{\partial D\left(E_{j}\right)}{\partial E_{j}}>0$ and $\frac{\partial^{2} D\left(E_{j}\right)}{\partial E_{j}{ }^{2}} \geq 0$. Given $D_{j}$, the socially efficient benchmark solution is determined via the benevolent social planner's problem,

$$
W=\left\{\begin{array}{l}
\operatorname{Max} \\
\left.v_{i j}\right\}_{i}
\end{array} \quad \sum_{i} B_{i}\left(v_{i}\right)-\sum_{i} \sum_{j}\left(c_{i}^{g} v_{i j}+c_{i}\left(v_{i j}\right)+F_{i j}\right)-\sum_{j} D\left(E_{j}\right)\right.
$$

resulting in the first-order efficiency conditions,

$$
\frac{\partial B_{i}\left(v_{i}\right)}{\partial v_{i j}}-c_{i}^{g}-\frac{\partial c_{i}\left(v_{i j}\right)}{\partial v_{i j}}-e_{i} \frac{\partial D\left(E_{j}\right)}{\partial E_{j}}=0, \quad \forall i, j
$$

which indicates that in the socially efficient solution household $i$ will choose its $v_{i j}$ up to the point where the private marginal benefit of VMT is equated with its social marginal cost on a daily basis for any given week. Social marginal cost is in turn divided between its private cost, $c_{i}^{g}+\frac{\partial c_{i}\left(v_{i j}\right)}{\partial v_{i j}}$, and external cost, $e_{i} \frac{\partial D\left(E_{j}\right)}{\partial E_{j}}$, components.

Denoting $v_{i j}^{*}$ and $E_{j}^{*}$, respectively, as the socially efficient levels of daily household VMT and aggregate emissions associated with (B1), and comparing equation (B1) with equation (A1), we 
see that $v_{i j}^{P}>v_{i j}^{*}$ and thus $E_{j}^{P}>E_{j}^{*}, i=1, \ldots, N$ and $j=1, \ldots, 5$. Comparing these two equations also indicates that a household- and day-specific Pigovian tax levied on the household's daily emissions level, $e_{i} v_{i j}$, would need to be set at the rate,

$$
t_{i j}^{*}=e_{i} \frac{\partial D\left(E_{j}^{*}\right)}{\partial E_{j}}
$$

Note that because $t_{i j}^{*}$ is levied directly on the household's daily emissions level, it is adjusted for the purchase of an additional vehicle solely via a corresponding revision to the household's $e_{i}$ value as the vehicle is incorporated into the household's fleet. 


\section{References}

Ajanovic, A., R. Haas, and F. Wirl (2016) 'Reducing $\mathrm{CO}_{2}$ emissions of cars in the EU: analyzing the underlying mechanisms of standards, registration taxes, and fuel Taxes', Energy Efficiency 9(4): 925-937.

Barter, P.A. (2005) 'A vehicle quota integrated with road usage pricing: a mechanism to complete the phase-out of high fixed vehicle taxes in Singapore', Transport Policy 12(6): 525-536.

Cao, J., X. Wang, and X. Zhong (2014) 'Did driving restrictions improve air quality in Beijing?', China Economic Quarterly 13(3): 1091-1126.

Cantillo V. and J.D.D. Ortuzar (2014) 'Restricting the use of cars by license plate numbers: a misguided urban transport policy', DYNA 81(188): 75-82.

Carnovale, M. and M. Gibson (2013) 'The effects of driving restrictions on air quality and driver behavior', Unpublished manuscript, UCSD Working Paper Series, Department of Economics, University of California, San Diego, [Available at] http://escholarship.org/uc/item/0v8813qm\#page-1.

Chen, Y. (2012) 'Transportation policy simulation and urban travel demand model', Master's Thesis, Peking University, Beijing, China.

Chen, Y., G.Z. Jin, N. Kumar, and G. Shi (2013) 'The promise of Beijing: evaluating the impact of the 2008 olympic games on air quality', Journal of Environmental Economics and Management 66: 424-443. 
Cropper, M.L., Y. Jiang, A. Alberini, and P. Baur (2013) 'Getting cars off the road: the costeffectiveness of an episodic pollution control program', Environment and Resource Economics 57(1): 117-143.

Cummings, R.G. and M.B. Walker (2000) 'Measuring the effectiveness of voluntary emission reduction programmes', Applied Economics 32(13): 1719-1726.

Cutter, W.B. and M. Neidell (2009) 'Voluntary information programs and environmental regulation: evidence from "spare the air"', Journal of Environmental Economics and Management 58(3): 253-265.

Davis. L.W. (2008) 'The effect of driving restrictions on air quality in Mexico City', Journal of Political Economy 116: 38-81.

eBejing.com (2015) 'Circular on rotation of tail number plate of no-driving vehicles in regional rush hours on working days', [Available at] http://www.ebeijing.gov.cn/Government/GovernmentBulletin/t1224982.htm.

Eskeland, G.S. and T. Feyzioglu (1997) 'Rationing can backfire: the 'day without a car' in Mexico City', The World Bank Economic Review 11(3): 383-408.

Feng, Y., D. Fullerton, and L. Gan (2013) 'Vehicle choices, miles driven, and pollution policies', Journal of Regulatory Economics 44(1): 4-29.

Fullerton, D. and S.E. West (2002) 'Can taxes on cars and on gasoline mimic an unavailable tax on emissions?', Journal of Environmental Economics and Management 43: 135-157. 
Fullerton, D. and S.E. West (2010) 'Tax and subsidy combinations for the control of car pollution', B.E. Journal of Economic Analysis and Policy 10(1): Article 8.

Gallego, F., J.P. Montero, and C. Salas (2013a) 'The effect of transport policies on car use: a bundling model with applications', Energy Economics 40: S85-S97.

Gallego, F., J.P. Montero, and C. Salas (2013b) 'The effect of transport policies on car use: evidence from Latin American cities', Journal of Public Economics 107: 47-62.

Giblin, S. and A. McNabola (2009) 'Modelling the impacts of a carbon emission-differentiated vehicle tax system on $\mathrm{CO}_{2}$ emissions intensity from new vehicle purchases in Ireland', Energy Policy 37: 1404-1411.

Guo, O. (2016) 'Want to drive in Beijing? Good luck in the license plate lottery', The New York Times. [Available at] https://www.nytimes.com/2016/07/29/world/asia/china-beijingtraffic-pollution.html?_r=2.

Hennessy, H. and R.S.J. Tol (2011) 'The impact of tax reform on new car purchases in Ireland." Energy Policy 39(11):7059-7067.

Henry, G.T. and C.S. Gordon (2003) 'Driving less for better air: impacts of a public information campaign', Journal of Policy Analysis and Management 22(1): 45-63.

Innes, R. (1996) 'Regulating automobile pollution under certainty, competition, and imperfect information', Journal of Environmental Economics and Management 31: 219-239.

Jiang, Y. (2009) 'Do people drive less on code red days?', Mimeo, Asian Development Bank, Manilla. 
Kerr, G.N. (1995) 'Managing congestion: economics of price and lottery rationing', Journal of Environmental Management 45(4): 347-364.

Kunert, U. and H. Kuhfeld (2007) 'The diverse structures of passenger car taxation in Europe and the EU Commissions proposal for reform', Transportation Policy 14: 306-316.

Liu, Y. and C. Cirillo (2015) 'Model system to evaluate impacts of vehicle purchase tax and fuel tax on household greenhouse gas emissions', Transportation Research Record 2503: 5159.

Mabit. S.L. and M. Fosgerau (2011) 'Demand for alternative-fuel vehicles when registration taxes are high', Transportation Research Part D-Transport and the Environment 16(3): 225-231.

National Conference of State Legislatures (NCSL) (2016) Registration and Title Fees By State. [Available at] http://www.ncsl.org/research/transportation/registration-and-title-feesby-state.aspx.

Osakwe, R. (2010) 'An analysis of the driving restriction implemented in San José, Costa Rica', Policy Brief, Environment for Development (EfD), [Available at] http://www.efdinitiative.org/publications/analysis-driving-restriction-implemented-sanjose-costa-rica.

Viard, V.B. and S. Fu (2013) 'The effect of Beijing's driving restriction on pollution and economic activity', Social Science Research Network (SSRN), [Available at] http://papers.ssrn.com/sol3/papers.cfm?abstract_id=1917110. 
Wang, L., J. Xu, X. Zheng, and P. Qin (2013) 'Will a driving restriction policy reduce car trips? A case study of Beijing, China', Discussion Paper Series, EfD DP 13-11, Environment for Development (EfD).

Wang, Y., J. Hao, M.B. McElroy, J.W. Munger, H. Ma, D. Chen, and C.P. Nielsen (2009) 'Ozone air quality during the 2008 olympics: effectiveness of emission restrictions', Atmospheric Chemistriy and Physics Discussions 9: 9927-9959.

West, S.E. (2004) 'Distributional effects of alternative vehicle pollution control policies', Journal of Public Economics 88(3-4): 735-757.

Wolff, H. (2014) 'Keep your clunker in the suburb: low emission zones and adoption of green vehicles', The Economic Journal 124: F481-F512.

Zhai, K. and T. Ying (2016) 'Beijing said to expand restrictions on vehicle use to fight smog', Bloomberg, [Available at] https://www.bloomberg.com/news/articles/2016-0108/beijing-said-to-expand-restrictions-on-vehicle-use-to-fight-smog.

Zhang, W., C.-Y.C.L. Lawell, and V.I. Umanskaya (2016) 'The effects of license plate-vased driving restrictions on air quality: theory and empirical evidence', Unpublished manuscript, University of California at Davis, [Available at] http://www.des.ucdavis.edu/faculty/Lin/driving_ban_paper.pdf. 
Figure 1. Effect of annual vehicle registration tax on probability of adverse scale effect.

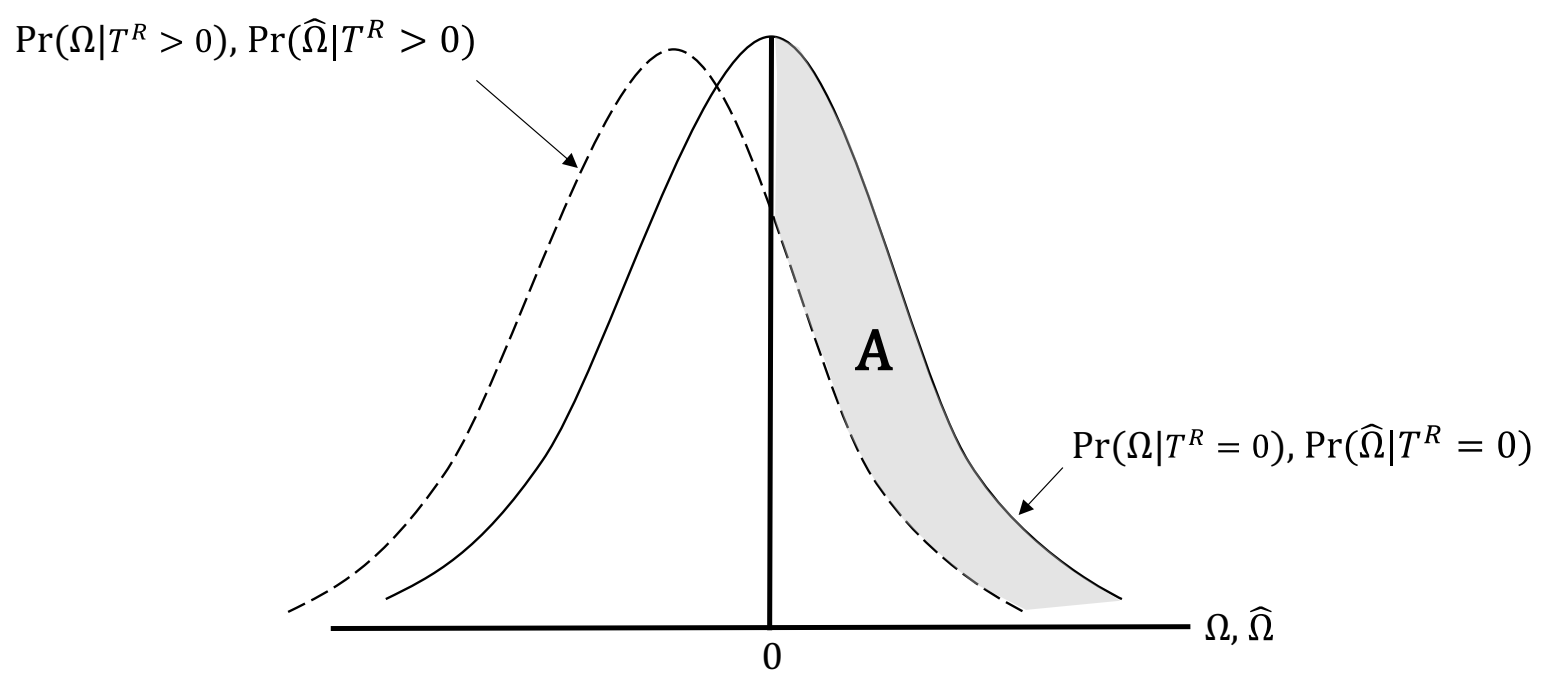


Table 1. Parameter values.

\begin{tabular}{|c|c|}
\hline Parameter & Value \\
\hline$I$ & 100 \\
\hline$\alpha_{1}$ & 7 \\
\hline$\alpha_{2}$ & 5 \\
\hline$p$ & 0.00005 \\
\hline$F_{1}$ & 0.005 \\
\hline$F_{2}$ & 0.005 \\
\hline$F^{A}$ & 0.005 \\
\hline$e$ & 1 \\
\hline
\end{tabular}


Table 2. Results.

\begin{tabular}{|c|c|}
\hline Variable & Value \\
\hline$v_{1}^{P}$ & 2.62 \\
\hline$v_{2}^{P}$ & 1.18 \\
\hline$v_{1}^{R}$ & 0 \\
\hline$v_{2}^{R}$ & 2.24 \\
\hline$E^{P}$ & 3.80 \\
\hline$E^{R}$ & 2.24 \\
\hline$\omega^{P}=E\left[\omega^{P}\right]$ & 102.83 \\
\hline$\omega^{R}=E\left[\omega^{R}\right]$ & 101.53 \\
\hline
\end{tabular}

